\title{
Comparison between Elastic-Perfectly-Plastic Interfacial Free-Edge and Crack Tip Singular Fields
}

\author{
Liviu Marsavina1,* - Andrew D. Nurse ${ }^{2}$ \\ 1 University Politehnica Timisoara, Romania \\ 2 Loughborough University, UK
}

The problem of the elastic and perfectly-plastic plane-strain asymptotic fields for the interfacial free-edge joint singularity is examined and compared with the interfacial crack tip. The geometries are idealised as isotropic elastic and elastic-perfectly-plastic materials bonded to a rigid elastic substrate. Under elastic behaviour it is difficult to directly compare the asymptotic fields between the joint and the crack due to the difference in singularity order and stress distributions. The elastic-perfectly-plastic fields for the interfacial free-edge joint were determined as polar stress components and as idealised plastic slipline sectors. A fourth-order Runge-Kutta numerical method provides solutions to fundamental equations of equilibrium and compatibility that are verified with those of a highly focused finite element (FE) analysis. A successful attempt to show that a direct comparison between the solutions for the interfacial free-edge joint and the Mode I interfacial crack exists if the deviatoric stresses are considered.

(C) 2011 Journal of Mechanical Engineering. All rights reserved.

Keywords: free-edge, crack tip, interface, elastic-perfectly-plastic

\section{INTRODUCTION}

Interface-controlled fracture is the initial microscopic event leading to ultimate macroscopic rupture in many polycrystalline and composite materials. Failure frequently initiates within the interfacial free-edge singularity being the source of unbounded elastic stresses and/or a plastic zone. The fracture process continues through propagation of an interface crack driven by complex stress intensity factors (SIFs). However, the actual events that take place between the former (initiation) and latter (propagation) are not well understood. From a design perspective, one may choose to prevent initial debonding of the interfacial joint or to prevent crack propagation. To add to the confusion though, it has been shown by Klingbeil and Bleuth [1] that conflicting solutions are obtained if designing to prevent debonding of the interfacial free-edge and subsequent propagation of the interfacial crack. Thus far, at least, it has been shown by Akinsaya and Fleck [2] that in the interim stage between initiation and propagation an interface crack has SIFs amplified if it is embedded in a free-edge singularity field. This may seem an issue related only to interfacial systems but considering that the vast majority of structural materials are either polycrystalline metals or composite materials, it is more fundamental than perhaps first considered.

One approach to understand the events that take place between crack initiation at the interfacial free edge and propagation of an interface crack would be to examine the change in stress state from the initiating stage to the final stage. However, to link the two fields in terms of elastic stresses and strains is problematic due to the general difference in singularity orders and resulting angular distributions. From an asymptotic perspective, the problem is incomprehensible given that one either has a free edge (Fig. 1a), or one has a crack (Fig. 1b); there is no in-between stage. Furthermore, most structural materials are also known to undergo, in general, small-scale yielding (SSY) before failure. Consequently, there seems little point in attempting to establish a direct link between crack initiation and propagation in the purely elastic regime. It seems more important to link the asymptotic fields of the interfacial free-edge joint and interfacial crack tip under elasto-plastic (SSY) conditions. Our understanding of the toughness of interfacial systems would be then increased with this link between the events of crack initiation, i.e. debonding of the interfacial free-edge joint and the propagation of an interfacial crack. 
In this paper, the structure of the asymptotic field at the interfacial free-edge is determined for an elastic-perfectly-plastic material bonded perfectly to a rigid elastic material forming a half plane. Stresses and displacements in the asymptotic fields are numerically calculated in an approach similar to Sharma and Aravas [3] and are verified by finite element (FE) analysis. To determine a pathological link between crack initiation at the interfacial free-edge and growth of an interface crack the two fields are compared directly. To compare for a range of possibilities the distributions of polar stress components are determined for the interfacial free-edge and crack-tip singular fields for the purely elastic and perfectly-plastic cases. In the latter case, direct comparisons are possible since the singularities are removed by material yielding except in the case of the radial shear strain. The plastic slipline field for the interface crack-tip characterized by Zywicz and Parks [4] is used to show that the interfacial free-edge joint solution is very similar to its counterpart crack-tip with a null elastic wedge sector.

a)

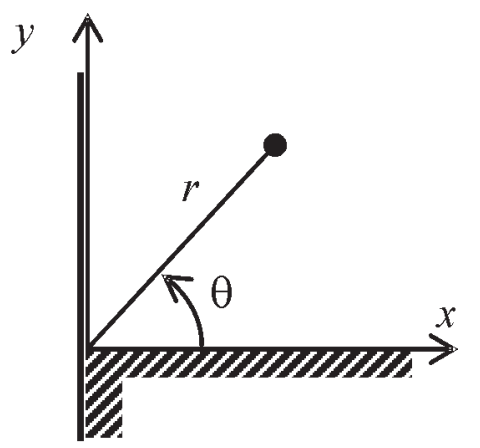

b)

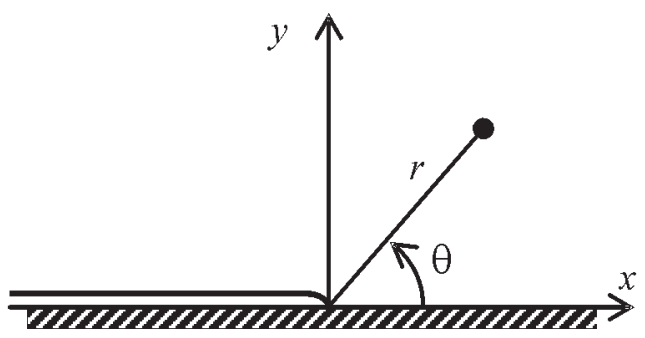

Fig. 1. Schematic a) interfacial free-edge joint and b) interfacial crack-tip geometries including polar and Cartesian co-ordinate systems

\section{THEORETICAL FORMULATION}

The singularity order of the interfacial crack tip under elastic conditions is always -0.5 in comparison to the free-edge joint that varies according to the elastic properties of the two materials. In general, the interface crack between two isotropic materials suffers a singularity stress field characterised by the complex SIF, $K=K_{1}+$ $i K_{2}$, and is of the form:

$$
\sigma_{i j}(r, \theta)=K r^{-0.5+\mathrm{i} \varepsilon} g_{i j}(\varepsilon, \theta),
$$

where $\varepsilon$ is the oscillatory index and $g_{i j}$ are known non-dimensional functions. A singularity at the interfacial free-edge is predicted by Bogy [5] to be of order depending on the elastic constants of the materials. For the interfacial free-edge joint the stress singularity corresponds to the form:

$$
\sigma_{i j}(r, \theta)=H r^{\lambda-1} f_{i j}(\lambda, \theta),
$$

where $H$ is its intensity, $\lambda-1$ is the singularity order, and $f_{i j}$ are known non-dimensional functions of $(\lambda, \theta)$. The order of the singularity $\lambda$ is dependent on the degree of material mismatch and the intensity $H$ depends on the far-field geometry and loading. For the purposes of this study the singularity order of the elastic case for an aluminium - epoxy joint $\left(E_{A l}=70000 \mathrm{MPa}, v_{A l}=0.33, E_{\text {epoxy }}=3000 \mathrm{MPa}\right.$, $\left.v_{\text {Epoxy }}=0.4\right)$ is $\lambda-1=-0.303$.

A fracture mechanics description of the critical state prior to separation using continuumbased mechanics usually involves the elastic solution for the crack. It is of limited use for describing materials that yield and undergo inelastic deformation at high strains. The elasto -plastic interfacial crack problem has received considerable attention in the last decade, enabling a thorough understanding to be developed. Using a $J$-integral argument Rice and Rosengren [6], and Hutchinson [7] have shown that crack problems under SSY conditions result in a $1 / r$ singularity in the strain-energy density and the radial shear strain fields. The analysis of interfacial free-edge is just as important to our understanding of crack initiation and growth though in comparison to its counterpart the interface crack it has received far less attention. It appears no effort has been made to understand the elasto-plastic behaviour of the free-edge singularity. The constitutive behaviour of a homogeneous isotropic elasto-plastic material 
may be characterised by the $J_{2}$ deformation theory for a Ramberg-Osgood uniaxial stress-strain behaviour, i.e.:

$$
\varepsilon_{i j}=\frac{1+v}{E} S_{i j}+\frac{1-2 v}{3 E} \sigma_{k k} \delta_{i j}+\frac{3}{2} \alpha \varepsilon_{\mathbf{0}}\left(\frac{\sigma_{e}}{\sigma_{\mathbf{o}}}\right)^{n-1} \frac{S_{i j}}{\sigma_{\mathbf{o}}},
$$

where $\varepsilon_{i j}$ is the infinitesimal strain tensor, $\sigma_{\mathrm{o}}$ is the yield stress, $\sigma_{e}$ is the Mises equivalent stress, $\mathrm{E}$ is the Young's moduli, $\varepsilon_{\mathrm{o}}=\sigma_{\mathrm{o}} / \mathrm{E}$ and $S_{i j}$ is the deviatoric stress. Also, $n$ is the power-law hardening exponent $(1 \leq n \leq \infty), \delta_{i j}$ is the Kronecker delta, and $\alpha$ is a material constant. Putting $n=\infty$ then the behaviour is elastic-perfectly-plastic.

Asymptotic solutions to the crack-tip behaviour under elastic-perfectly-plastic conditions may be obtained in polar co-ordinate form $(r, \theta)$ using the expansion form given by Sharma and Aravas [3]. To obtain the asymptotic solution the problem is formulated in terms of the leading order stresses $\tilde{\boldsymbol{\sigma}}^{(0)}$ and displacements $\mathbf{u}^{(0)}$ that are substituted into the governing equations of equilibrium, compatibility, and stress-strain relationship. Terms having like powers of radius $r$ are collected and hierarchy of problems is obtained. The leading order problem that defines $\boldsymbol{\sigma}^{(0)}$ and $\mathbf{u}^{(0)}$ consists of five non-linear ordinary differential equations, where $s$ is the stress singularity order [8].

The focus of the paper is the leading order solution for the interfacial free-edge joint and its similarities with that of the crack tip where $s=-1 /(1+n)$.

$$
\begin{aligned}
&(s+1) \tilde{\sigma}_{r r}^{(0)}-\tilde{\sigma}_{\theta \theta}^{(0)}+\frac{d \tilde{\sigma}_{r \theta}^{(0)}}{d \theta}=0,=\frac{d \tilde{\sigma}_{\theta \theta}^{(0)}}{d \theta}+(s+2) \tilde{\sigma}_{r \theta}^{(0)}=0, \\
&(s n+1) \tilde{u}_{r}^{(0)}-\frac{3}{2} \tilde{\sigma}_{e}^{(0) n-1} \tilde{S}_{r r}^{(0)}=0, \\
& \tilde{u}_{r}^{(0)}+\frac{d \tilde{u}_{\theta}^{(0)}}{d \theta}-\frac{3}{2} \tilde{\sigma}_{e}^{n-1} \tilde{S}_{\theta \theta}^{(0)}=0, \\
& \frac{1}{2}\left(\frac{d \tilde{u}_{r}^{(0)}}{d \theta}+\operatorname{snn} \tilde{u}_{\theta}^{(0)}\right)-\frac{3}{2} \tilde{\sigma}_{e}^{(0) n-1} \tilde{\sigma}_{r \theta}^{(0)}=0 .
\end{aligned}
$$

\section{THE DEVELOPMENT OF THE ELASTIC- PERFECTLY-PLASTIC FIELD FOR THE INTERFACIAL FREE-EDGE JOINT AND BIMATERIAL INTERFACE CRACK}

A fourth-order Runge-Kutta solution to the Eq. (3) was obtained for an elastic-perfectlyplastic hardening exponent $(n>1000)$, using the proprietary software Mathcad (v.2000), distributed by Adept Scientific Ltd. $n$ iteration scheme was used to determine the solution $s$ to the non-linear eigenvalue problem and the subsequent distributions for the stresses and displacements that satisfy the governing equations and imposed boundary conditions, [8]. This asymptotic solution was verified by a FE analysis performed by using the software Lusas (v13.3, distributed by FEA Ltd., UK). Highly-focused, refined meshes for the interfacial free-edge joint and bimaterial interface crack geometries were prepared using
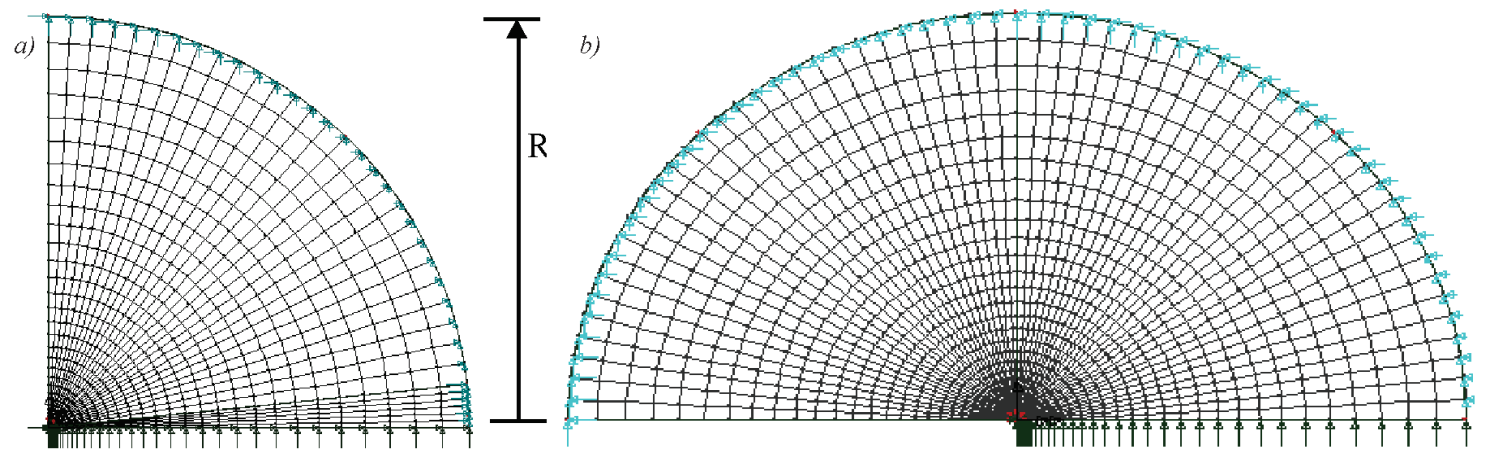

Fig. 2. Mesh and boundary condition for the a) interfacial free-edge joint (2074 elements, 2136 nodes) and b) interfacial crack-tip models (4500 elements, 4651 nodes) 
four-noded linear elements until satisfactory convergent results were obtained. The boundary conditions were defined as applied displacements corresponding to the elastic solution for the model radius $R=5 \mathrm{~mm}$, and a perfect bond to the rigid substrate was defined, Fig. 2.
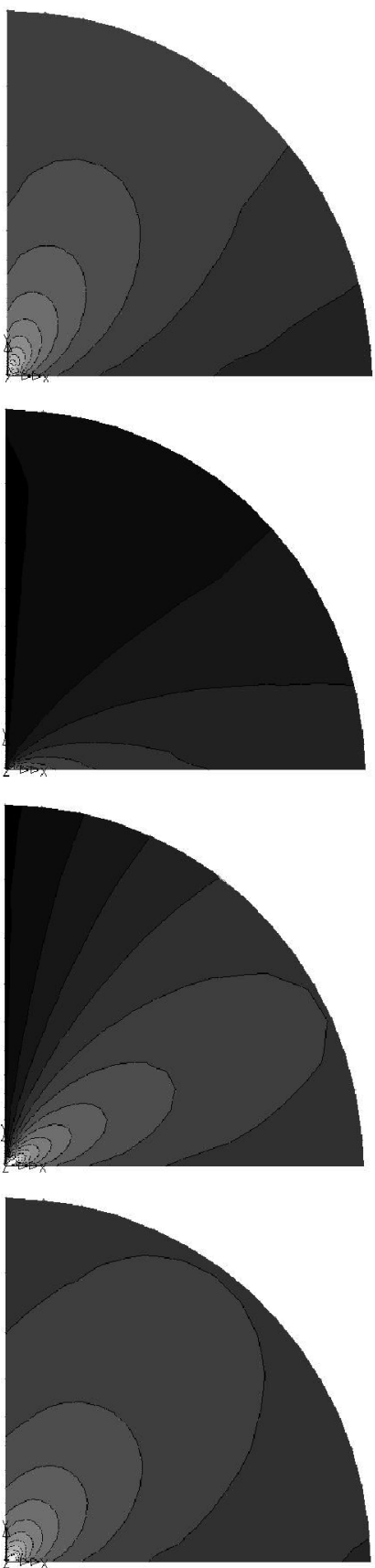
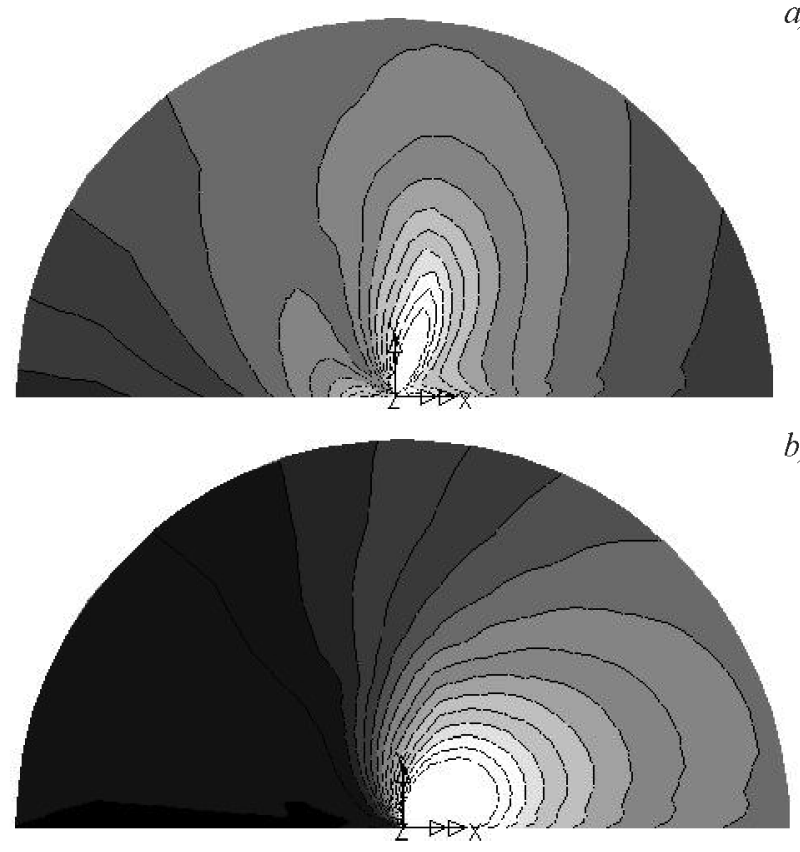

b)
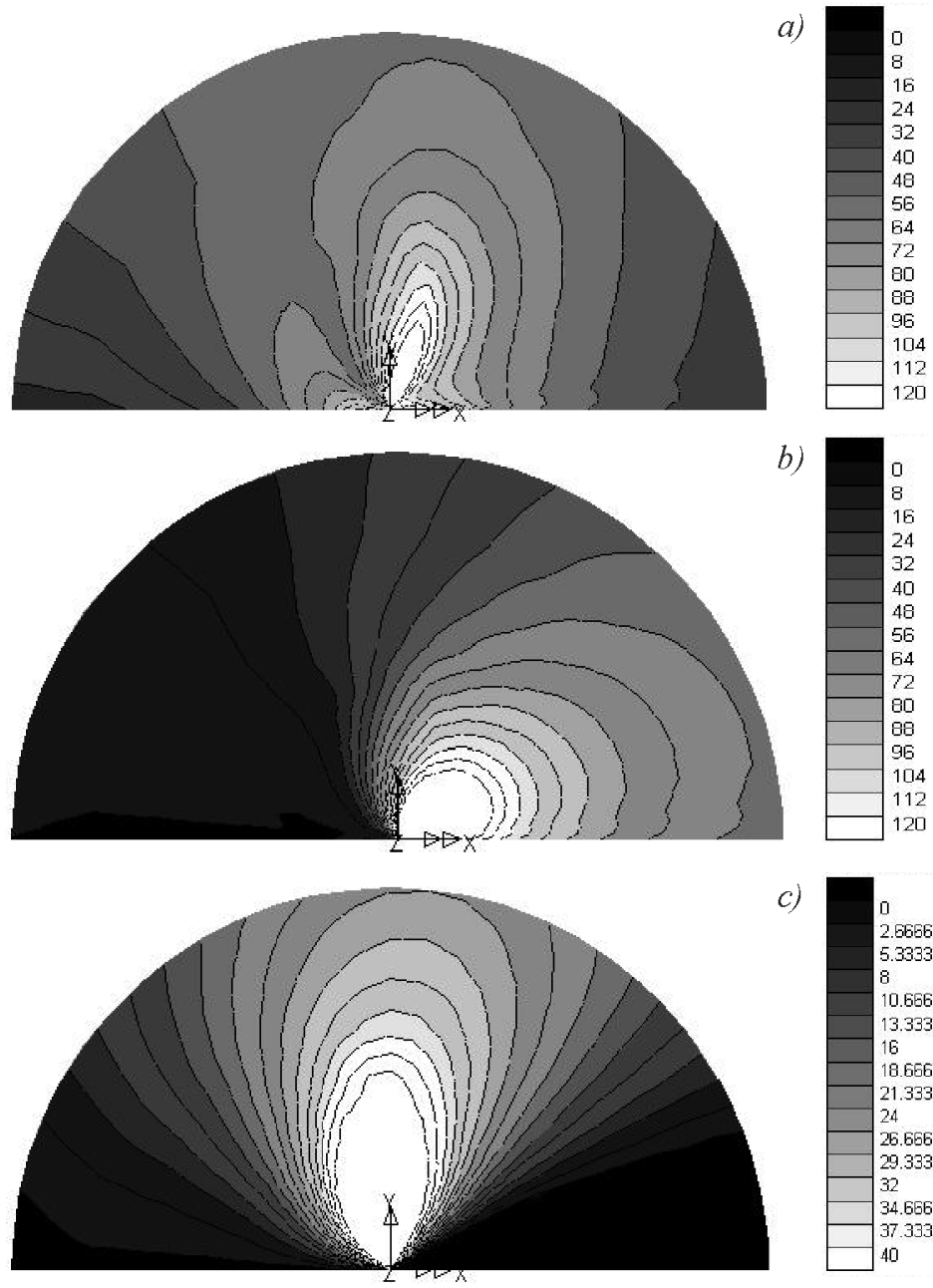

c)

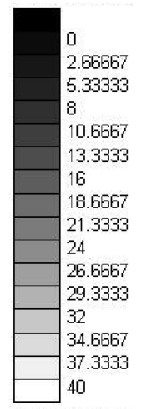

d)
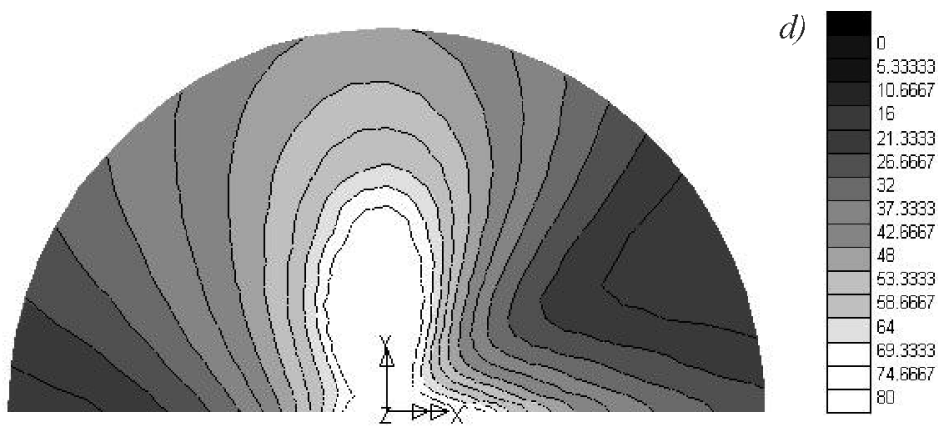

Fig. 3. Contour plots showing results of a FE analysis for the elastic-perfectly-plastic upper region of an interfacial free-edge joint and crack tip bonded to a rigid elastic lower region; a) $s_{x x}$ for interfacial free-edge and interfacial crack tip (in MPa), b) $s_{y y}$ for interfacial free-edge and interfacial crack tip (in $M P a), c) s_{x y}$ for interfacial free-edge and interfacial crack tip (in MPa), d) $s_{e}$ for interfacial free-edge and interfacial crack tip (in $\mathrm{MPa}$ ) 
The results of the FE analysis for interfacial free-edge joint and crack tip under SSY elasticperfectly-plastic conditions are shown in Fig. 3 as contour plots for the region $-10^{-5} \leq r / r_{p} \leq 10^{-3}$ where $r_{p}$ is the maximum extent of the plastic zone defined by $\sigma_{e}=\sigma_{o}$. The results for the Cartesian stress components $\sigma_{i j}$, and for the equivalent stress $\sigma_{e}$ are plotted using the same scale for interfacial free-edge joint and crack tip.

Fig. 4a shows the angular variation of the polar components of stress for the interfacial freeedge joint. Nodal values of the polar components of stress from the FE analysis are plotted as symbols for the radius $\log \left(r / r_{p}\right)=-2$. The results are normalised so that the maximum value of the equivalent stress $\sigma_{e}$ in the angular variation is unity, i.e.:

$$
\left(1.5 \tilde{S}_{i j}^{(0)} \tilde{S}_{i j}^{(0)}\right)_{\max }^{1 / 2}=1 .
$$

The fourth-order Runge-Kutta solution to the angular variations satisfying (3) and the imposed boundary conditions has been superimposed onto the FE results to enable them to be validated, [9]. In all cases of the polar components of stress, the agreement between the asymptotic solution and the FE results is excellent.

The rigid-slip-line field solution for the interfacial free-edge may be characterised using the notation of Zywicz and Parks [4]. The three inelastic crack-tip sectors admissible are the

a)

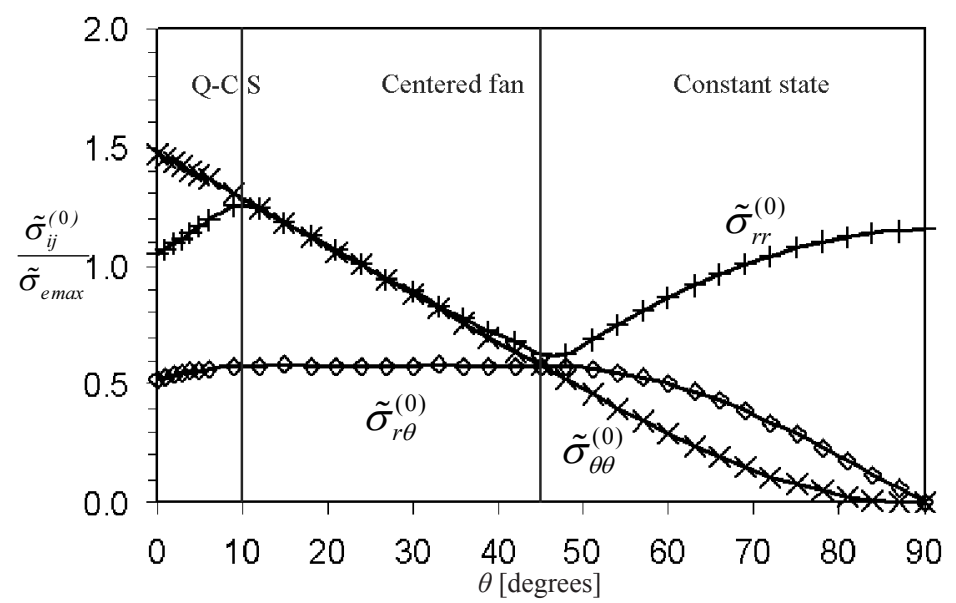

centred fan, constant-state sector, and the quasiconstant-state sector [4]. Elastic crack-tip sectors are also admissible and asymptotically they behave as semi-infinite elastic wedges loaded by constant surface tractions. The solution for the plastic slip-line field was determined and is shown in Fig. 4b. A quasi-constant-state sector exists at the interface followed by comparatively large angular distributions of centred fan and constant state. A characteristic of the centred fan region is a singularity in the shear strain and the order was found to be approximately -0.8 (c.f. -1 for the crack).

\section{COMPARISON BETWEEN INTERFACIAL FREE-EDGE JOINT AND CRACK TIP ASYMPTOTIC FIELDS}

Figs. 5a and $\mathrm{b}$ show the normalised solutions for the polar and deviatoric stress components for the two geometries assuming purely elastic behaviour. Asymptotic solutions assuming elastic perfectly-plastic behaviour that satisfy (3), were produced for both geometries under remote Mode I loading. It was considered here that there may be some similarity between the inelastic behaviour at the interfacial freeedge joint and the Mode I interfacial crack since the latter case includes an elastic sector between $90^{\circ} \leq \theta \leq 180^{\circ}$, [4]. That is, both are inelastic between $0^{\circ} \leq \theta \leq 90^{\circ}$ and the plasticity is confined to the same quarter. The normalised results for

b)

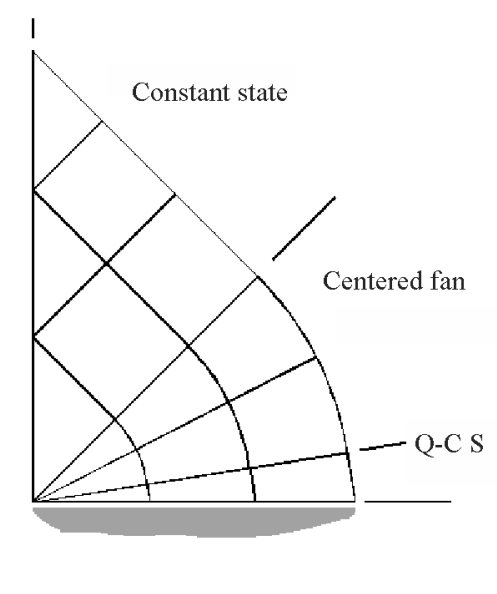

Fig. 4. Rigid-slip-line field solution at an interfacial free-edge singularity for an elastic-perfectly-plastic upper region bonded to a rigid elastic substrate; a) the angular variation of the polar components of stress for the interfacial free-egde joint; $b$ ) the solution for the plastic slip-line field 

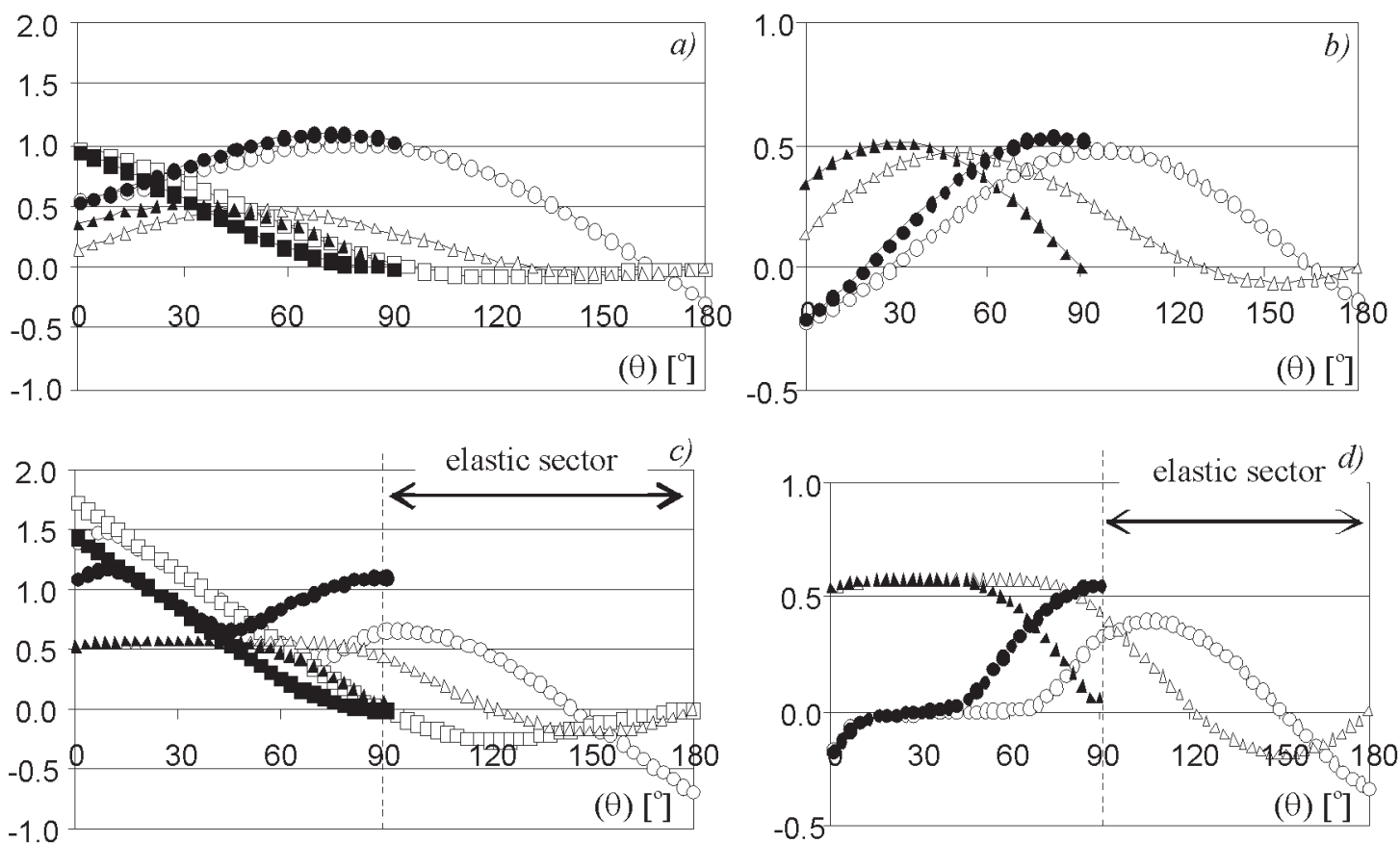

Legend:

- $-\tilde{\sigma}_{\theta \theta}^{(0)}, \bullet-\tilde{\sigma}_{r r}^{(0)}, \mathbf{\Delta}-\tilde{\sigma}_{r \theta}^{(0)}$ for interfacial free-edge

- $-\tilde{S}_{r r}^{(0)}, \boldsymbol{\Delta}-\tilde{S}_{r \theta}^{(0)}$ for interfacial free-edge

$\square-\tilde{\sigma}_{\theta \theta}^{(0)}, \bigcirc-\tilde{\sigma}_{r r}^{(0)}, \triangle-\tilde{\sigma}_{r \theta}^{(0)}$ for interfacial crack tip

$\bigcirc-\tilde{S}_{r r}^{(0)}, \triangle-\tilde{S}_{r \theta}^{(0)}$ for interfacial crack tip

Fig. 5. Comparison of the asymptotic normalised plane-strain stress components for the interfacial freeedge and crack-tip under elastic-perfectly-plastic slip conditions; a) polar elastic stress components, b) deviatoric elastic stress components, c) polar perfectly-plastic stress components, d) deviatoric perfectlyplastic stress components

the polar components of stress for the interfacial crack and those for the free-edge joint are shown together in Fig. 5c to enable comparisons to be made. The "elastic sector" indicated is the solution for a $90^{\circ}$ elastic wedge and not those of Eq. (3).

For the elastic solutions, there is some similarity between the forms of the polar components of stress for the two geometries between $0^{\circ}$ and $90^{\circ}$. The deviatoric stresses demonstrate, however, that there are still some major differences between the two geometries; it should also be emphasised that the singularity orders are -0.28 and -0.5 for the interfacial free-edge joint and crack tip, respectively. Under perfectly-plastic behaviour, instead, the singularity orders are -0.8 and -1 for shear strain for the interfacial free-edge joint and crack tip, respectively. There is no singularity for the stresses in both cases. The resulting distributions for the polar components of stress shown in Fig. 5c compare very favourably in form. A more obvious similarity is obtained when the deviatoric stresses are plotted as in Fig. 5d. Here, the two sets of curves cannot be distinguished for the region $0^{\circ} \leq \theta \leq 45^{\circ}$. It may be surmised that upon development of an interfacial crack from an interfacial free-edge joint the asymptotic deviatoric stress field does not have to change in the region $0^{\circ} \leq \theta \leq 45^{\circ}$ for an elastic-perfectlyplastic material. In other words, upon attaining yielding stress levels a 'pseudo' crack-tip will exist at the interfacial free-edge that has the same asymptotic field for the deviatoric stresses as for the interfacial crack. The close similarity ends at $\theta=45^{\circ}$ where the slip-line sectors for the joint undergo a transition from centered fan to constant 
state whereas for the crack this takes place at about $60^{\circ}$.

\section{CONCLUSIONS}

The plastic-slip-line field has been sectorally assembled for elastic-perfectly-plastic behaviour at the interfacial free-edge joint. This is found to be in agreement with a Mode I solution for the interfacial crack. In comparing stress field solutions for the interfacial free-edge joint with that of the crack, involving an elastic sector between $90^{\circ} \leq \theta \leq 180^{\circ}$, it is seen that the normalised deviatoric stress field is indistinguishable between the two geometries for the region $0^{\circ} \leq \theta \leq 45^{\circ}$. Consequently, it would appear that the asymptotic deviatoric stress field that drives many forms of crack propagation does not need to change structure upon initiation of an interfacial crack at the free-edge joint.

\section{ACKNOWLEDGEMENTS}

Financial support from the European Commision trough programm FP7-PEOPLEERG-2007 under grant agreement no. 230991 is gratefully acknowledged.

\section{REFERENCES}

[1] Klingbeil, N.W., Beuth, J.L. (2000). On the design of debonding - resistant Bimaterials. Part I: Free-edge singularity approach. Engng. Fract. Mech., vol. 66, p. 93-110.
[2] Akisanya, A.R., Fleck, N.A. (1997). Interfacial cracking from the free-edge of a long bi-material strip. Int. J. Solids Structures, vol. 34, p. 1645-1665.

[3] Sharma, S.M., Aravas, N. (1991). Determination of higher order terms in asymptotic elastoplastic crack tip solutions. J. Mech. Phys. Solids, vol. 39, p. 1043-1072.

[4] Zywicz, E., Parks, D.M. (1992). Small-scale yielding interfacial crack-tip fields. $J$. Mech. Phys. Solids, vol. 40, p. 511-536.

[5] Bogy, D.B. (1971). Two edge-bonded elastic wedges of different materials and wedge angles under surface traction. J. Appl. Mechs., vol. 38, p. 377-386.

[6] Rice, J.R., Rosengren, G.R. (1968). Plane strain deformation near a crack tip in a power - law hardening material. J. Mech. Phys. Solids, vol. 16, p. 1-12.

[7] Hutchinson, J.W. (1968). Singular behaviour at the end of a tensile crack in a hardening material. J. Mech. Phys. Solids, vol. 16, p. 13-31.

[8] Marsavina, L., Nurse, A.D. (2007). The asymptotic structure of small-scale yielding interfacial free-edge joint and crack-tip fields. Acta Mechanica, vol. 190, p. 115131.

[9] Marsavina, L., Nurse, A.D. (2003). Similarities between small-scale yielding interfacial free-edge joint and crack-tip fields. Facta Universitatis, vol. 3, no. 13, p. 623-634. 